\title{
Recombination dynamics of a localized exciton bound at basal stacking faults within the $\mathrm{m}$ - (p)lane $\mathrm{ZnO}$ film
}

S. Yang, H. C. Hsu, W-R. Liu, B. H. Lin, C. C. Kuo, C-H. Hsu, M.O. Eriksson, Per-Olof Holtz and W. F. Hsieh

\section{Linköping University Post Print}

\section{Tweet}

N.B.: When citing this work, cite the original article.

Original Publication:

S. Yang, H. C. Hsu, W-R. Liu, B. H. Lin, C. C. Kuo, C-H. Hsu, M.O. Eriksson, Per-Olof Holtz and W. F. Hsieh, Recombination dynamics of a localized exciton bound at basal stacking faults within the m-(p)lane $\mathrm{ZnO}$ film, 2014, Applied Physics Letters, (105), 1, 011106.

http://dx.doi.org/10.1063/1.4887280

Copyright: American Institute of Physics (AIP)

http://www.aip.org/

Postprint available at: Linköping University Electronic Press

http://urn.kb.se/resolve?urn=urn:nbn:se:liu:diva-109886 


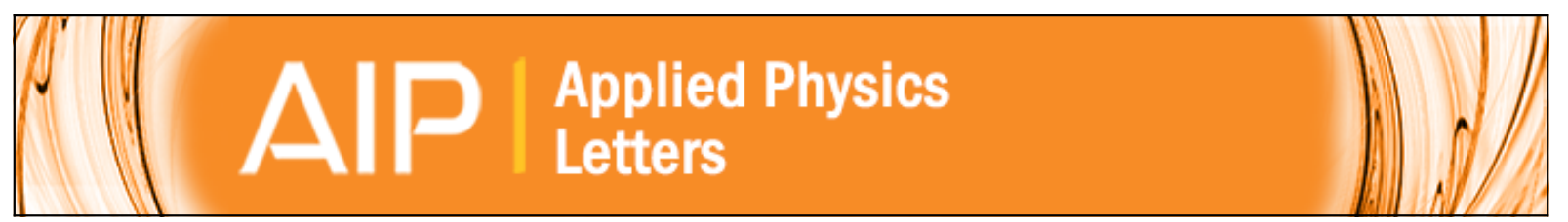

Recombination dynamics of a localized exciton bound at basal stacking faults within the m-plane $\mathrm{ZnO}$ film

S. Yang, H. C. Hsu, W.-R. Liu, B. H. Lin, C. C. Kuo, C.-H. Hsu, M. O. Eriksson, P. O. Holtz, and W. F. Hsieh

Citation: Applied Physics Letters 105, 011106 (2014); doi: 10.1063/1.4887280

View online: http://dx.doi.org/10.1063/1.4887280

View Table of Contents: http://scitation.aip.org/content/aip/journal/apl/105/1?ver=pdfcov

Published by the AIP Publishing

\section{Articles you may be interested in}

Photoluminescence associated with basal stacking faults in c-plane $\mathrm{ZnO}$ epitaxial film grown by atomic layer deposition

Appl. Phys. Lett. 100, 101907 (2012); 10.1063/1.3692730

Exciton localization on basal stacking faults in a -plane epitaxial lateral overgrown GaN grown by hydride vapor phase epitaxy

J. Appl. Phys. 105, 043102 (2009); 10.1063/1.3075596

Complex excitonic recombination kinetics in $\mathrm{ZnO}$ : Capture, relaxation, and recombination from steady state Appl. Phys. Lett. 90, 041917 (2007); 10.1063/1.2432259

Radiative and nonradiative recombination processes in lattice-matched ( $\mathrm{Cd}, \mathrm{Zn}) \mathrm{O} /(\mathrm{Mg}, \mathrm{Zn}) \mathrm{O}$ multiquantum wells Appl. Phys. Lett. 77, 1632 (2000); 10.1063/1.1308540

Radiative recombination centers induced by stacking-fault pairs in ZnSe/ZnMgSSe quantum-well structures Appl. Phys. Lett. 75, 3944 (1999); 10.1063/1.125502

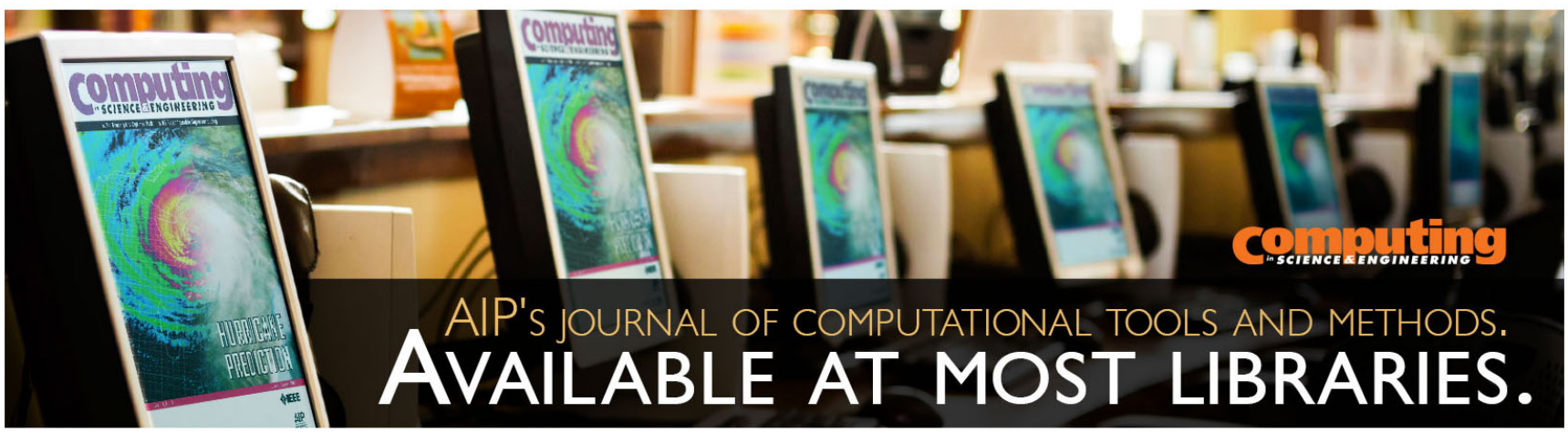




\title{
Recombination dynamics of a localized exciton bound at basal stacking faults within the $\boldsymbol{m}$-plane $\mathrm{ZnO}$ film
}

\author{
S. Yang, ${ }^{1}$ H. C. Hsu, ${ }^{2, a)}$ W.-R. Liu, ${ }^{1}$ B. H. Lin,,${ }^{1,3}$ C. C. Kuo, ${ }^{3}$ C.-H. Hsu, ${ }^{1,3}$ M. O. Eriksson, ${ }^{4}$ \\ P. O. Holtz, ${ }^{4}$ and W. F. Hsieh ${ }^{3, a)}$ \\ ${ }^{1}$ Scientific Research Division, National Synchrotron Radiation Research Center, Hsinchu 30076, Taiwan \\ ${ }^{2}$ Department of Photonics and Advanced Optoelectronic Technology Center, National Cheng Kung University, \\ 701 Tainan, Taiwan \\ ${ }^{3}$ Department of Photonics and Institute of Electro-Optical Engineering, National Chiao Tung University, \\ Hsinchu 30010, Taiwan \\ ${ }^{4}$ Department of Physics, Chemistry and Biology (IFM), Linköping University, Linköping, Sweden
}

(Received 12 March 2014; accepted 24 June 2014; published online 7 July 2014)

\begin{abstract}
We investigated the carrier dynamics near basal stacking faults (BSFs) in $m$-plane $\mathrm{ZnO}$ epitaxial film. The behaviors of the type-II quantum wells related to the BSFs are verified through timeresolved and time-integrated photoluminescence. The decay time of the emission of BSFs is observed to have a higher power law value and longer decay time than the emission of the donorbound excitons. The spectral-dependent decay times reveal a phenomenon of carriers migrating among band tail states, which are related to the spatial distribution of the type-II quantum wells formed by the BSFs. A high density of excited carriers leads to a band bending effect, which in turn causes a blue-shift of the emission peak of BSFs with a broadened distribution of band tail states. C 2014 AIP Publishing LLC. [http://dx.doi.org/10.1063/1.4887280]
\end{abstract}

$\mathrm{ZnO}$, a wide direct band gap material, has attracted much attention due to its potential for applications in ultraviolet photonic devices. ${ }^{1-4}$ The presence of the built-in electric field in $c$-plane-grown $\mathrm{ZnO}$ reduces the emission efficiency. To avoid this problem, much research has been focused on the growth of non-polar ( $a$ - or $m$-planes) $\mathrm{ZnO}$ epitaxial films. Since $\mathrm{ZnO}$ substrates are very rare and expensive, other foreign substrates, such as sapphire, $\mathrm{SiC}$, and $\mathrm{LaAlO}_{3}$, are the most commonly used substrates to grow nonpolar $\mathrm{ZnO}$ and related alloys. Therefore, this heteroepitaxy results in the generation of a high density of structural defects, such as dislocations and stacking faults. The heteroepitaxial non-polar epitaxial films suffer from a high density of basal stacking faults (BSFs). From the viewpoint of crystal growth, defects such as BSFs should be eliminated or at least significantly reduced. Several growth methods have been proposed to reduce the density of BSFs in $\mathrm{ZnO}$ and IIINitride materials. ${ }^{5}$ In contrast, some of these defects have exposed unusual properties, which have potential to be employed in particular applications. Interestingly, BSFs exhibiting optical activity strongly influence the luminescence properties of the materials with wurtzite structure such as $\mathrm{GaN}$ and $\mathrm{ZnO} .^{6-10}$ Through $a b$ initio calculations, the $\mathrm{BSF}$ is predicted to have the zinc-blend (ZB) lattice structure embedded in the wurtzite (WZ) $\mathrm{ZnO}$ matrix, which forms a type-II quantum well (QW). ${ }^{11}$ Besides possible applications as lighting devices, the type-II QW structures in semiconductor materials are promising for photovoltaic devices as a result of their spatial separation of photoexcited electrons and holes. ${ }^{12,13}$ Consequently, this ZnO-based spontaneously formed type-II QW system has become alternatively important. Although it is essential to understand the luminescence

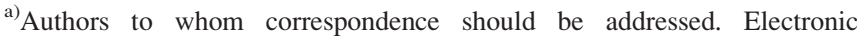
addresses: hsuhc@mail.ncku.edu.tw and wfhsieh@mail.nctu.edu.tw
}

mechanism and to investigate the photoexcited carrier dynamics, published reports on the experimental aspects of the carrier dynamic properties of this material system have been limited up to now. In this article, we present a detailed emission mechanism of the BSFs in the $m$-plane $\mathrm{ZnO}$ layers. We report on some peculiar behaviors such as S-shaped peak shifting due to the composition fluctuation and the migration of photoexcited carriers among the band tail states. The high density of electrons accumulated at the interface between the potential well and barrier result in a band bending effect that broadens the distribution of these energy levels and decreases the decay times.

An epitaxial $m$-plane $\mathrm{ZnO}$ layer with a thickness of $130 \mathrm{~nm}$ was grown on $m$-plane sapphire substrates by the atomic layer deposition (ALD) method (for details, see Ref. 14). The TEM results show that BSFs are the predominant defect with a density of $\sim 10^{6} \mathrm{~cm}^{-1}$. For the transient photoluminescence (PL) measurements, the excitation from the third harmonic $\left(\lambda_{\text {exc }}=266 \mathrm{~nm}\right)$ of a Ti:sapphire femtosecond pulsed laser (pulse width $150 \mathrm{fs}$ ) with the repetition rate of $76 \mathrm{MHz}$ was employed, and the laser spot was focused down to about $1 \mu \mathrm{m}$. The time-resolved PL (TR-PL) signal was detected by a UV-sensitive Hamamatsu streak camera with a temporal resolution better than 20 ps.

The low-temperature time-integrated PL (TI-PL) spectra of the sample with different excitation powers are shown in Figure 1(a). The dominating emission at $3.32 \mathrm{eV}$ results from the BSFs, and the emission centered at $3.37 \mathrm{eV}$ is attributed to the near-band-edge (NBE) emission bands consisting of the donor-bound exciton $\left(\mathrm{D}^{0} \mathrm{X}\right)$ and the free exciton $(\mathrm{FX}){ }^{7}$ The $\mathrm{D}^{\circ} \mathrm{X}$ emission has a rather broad width $(\mathrm{FWHM} \sim 22 \mathrm{meV})$ as compared with those of the pulsed-laser-deposition-grown $\mathrm{ZnO}$ films (FWHM $\sim 10 \mathrm{meV}$ and typical $9-15 \mathrm{meV}){ }^{15,16}$ Some of the literature ${ }^{17-19}$ has reported that $\mathrm{D}^{\mathrm{o}} \mathrm{X}$ emissions with a lifetime range from 230 to 370 ps were observed in 

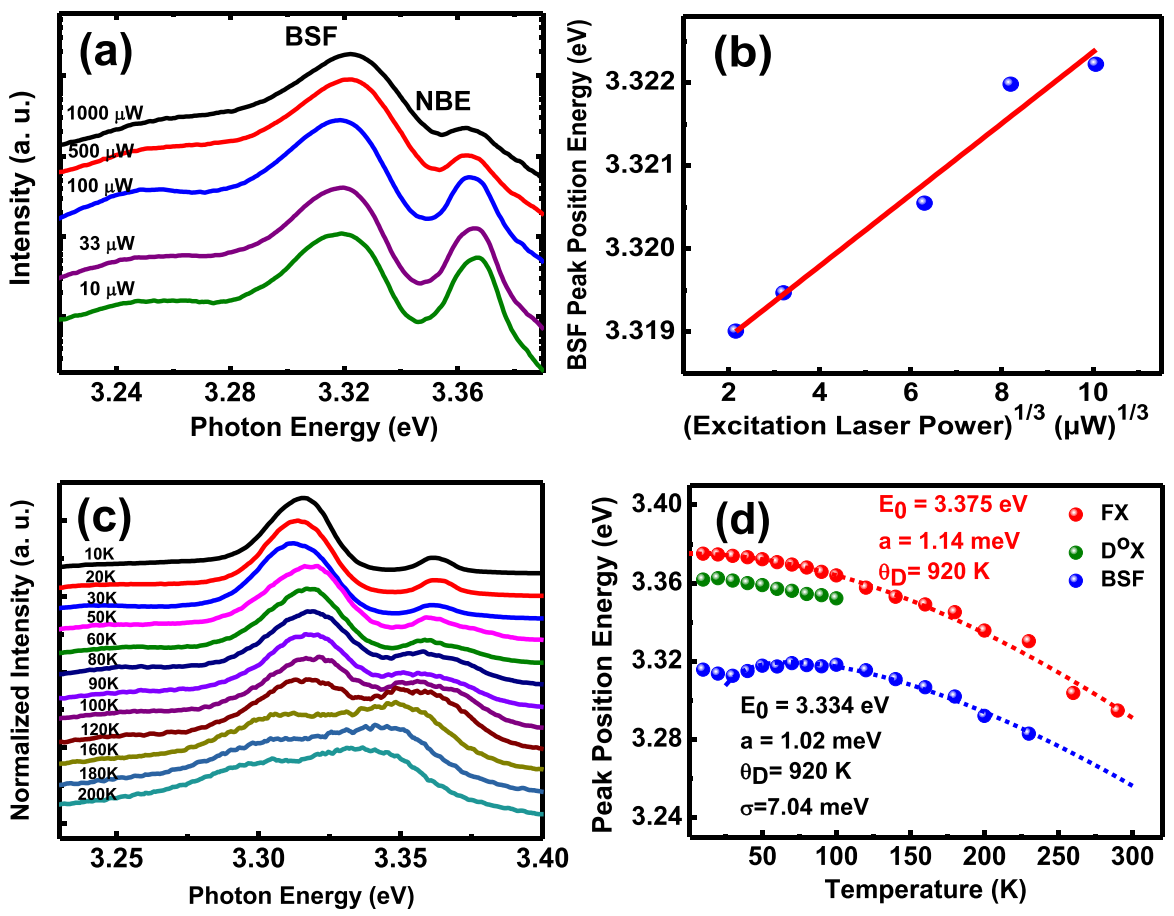

FIG. 1. (a) Power dependent TI-PL spectra recorded at $5 \mathrm{~K}$ of the $\mathrm{ZnO}$ film grown by ALD and annealed at $600^{\circ} \mathrm{C}$. (b) The intensity versus the excitation power of the BSF and NBE emissions. The dashed lines represent the outcome of the power-law fitting work. (c) The temperature dependent TI-PL spectra of the $\mathrm{ZnO}$ film taken between 10 and $200 \mathrm{~K}$. (d) The energy versus temperature plot of the BSF and NBE emissions. The dashed lines depict the fitting results to the Varshni's law. high-quality $\mathrm{ZnO}$ thin film, with a line width of less than $5 \mathrm{meV}$. Compared to these reported results, the rather large line width and short lifetime of the $\mathrm{D}^{\circ} \mathrm{X}$ emission in this paper could be due, we believe, to defects such as Zinc vacancy and/or BSFs, which may have enhanced the non-radiative transition from the $\mathrm{D}^{\circ} \mathrm{X}$. The energy peak of the BSF emission reveals a blueshift from 3.319 to $3.322 \mathrm{eV}$ as the excitation power is increased, and the peak energy as a function of the excitation power is shown in Figure 1(b). In type-II heterostructures of other material systems, a blueshift of the peak energy due to excitation power increment has been reported. ${ }^{20,21}$ In our case, the electrons and holes are spatially separated in different regions; that is, the electrons are confined in the $\mathrm{ZB}$ structure of the $\mathrm{ZnO}$ layer, and the holes are localized in the $\mathrm{WZ}$ structure of the $\mathrm{ZnO}$ layer. The well width formed by BSFs is $0.8 \mathrm{~nm}$ (exactly 1.5 times that of the c-axis lattice constant $)^{9}$ less than the exciton Bohr radius of $2.3 \mathrm{~nm}$, which leads to the zero Stokes-shift that originated from the screening effect. ${ }^{22,23}$ Therefore, the blueshift for increasing excitation power may not be a result of screening the electric fields. Such spatial separation between electrons and holes leads to an electric field, which gives rise to the bending of the valence and conduction band. The electrons and holes accumulated at the interface result in the formation of the potential well, which can be treated as approximately triangular. Thus, the electron quantization energy is expected to be proportional to the third root of the excitation power. ${ }^{24}$ As shown in Figure 1(b), the PL peak position energy can be fitted well by the third root of the excitation power, which indicates that the blueshift indeed originates from the recombination in the type-II QWs. ${ }^{25,26}$

In Figure 1(c), the energy peak of the BSF emission (marked by solid black circles) has a peculiar shift with raising temperature: the peak energy as a function of the temperature is shown in Figure 1(d) and termed as a "S-shape behavior," which is attributed to the localization effect observed on the excited carriers in the QWs. ${ }^{27}$ The S-shape behavior can be explained by band filling in the tails of the density-of-states (DOS), which implies that band tail states exist in the $m$-plane $\mathrm{ZnO}$ thin film. The model assumes that there are a certain amount of trap states forming the band tails with the DOS of Gaussian distribution, and the temperature-dependent peak energy is given by ${ }^{28}$

$$
h \nu_{0}=E_{0}-\frac{\sigma^{2}}{k_{B} T},
$$

where $E_{0}$ is the transition energy determined by the centers of the DOS Gaussian distributions of the electrons in the band tail and the holes in the valance band, and $\sigma^{2} / k_{B} T$ is the Stokes-type redshift, and where $\sigma$ is the dispersion of the Gaussian of the band tail, which is correlated to the localization energy. The quantity $E_{0}$ relates to the band gap described by Varshni's equation; therefore, the temperaturedependent equation of the peak energy can be described as

$$
E_{0}(T)=E_{0}(0)-\frac{\alpha T^{2}}{T+\beta}-\frac{\sigma^{2}}{k_{B} T} .
$$

From the fitting of the S-shaped curve of the BSF peak energy as a function of the temperature to Eq. (3) (see Fig. $1(\mathrm{~d})$ ), the parameter values of $E_{0}(0) \approx 3.334 \mathrm{eV}$, $\alpha \approx 1.02 \mathrm{meV}, \beta \approx 920 \mathrm{~K}$, and $\sigma \approx 7.04 \mathrm{meV}$ are obtained. The fitting results reveal that the BSF emission at low temperatures originates from the transition between the electrons trapped in the QWs formed by the BSFs and the holes in the valance band. Then the electrons in the QWs are further trapped into the band tail states. Subsequently, the electrons in the QWs and the holes in the valance band are considered to form BSFbound excitons, which are further confined into the band tail states. The NBE emission is composed of the $\mathrm{D}^{\circ} \mathrm{X}$ (marked by hollow red circles) and the FX (solid red circles) transitions. In 

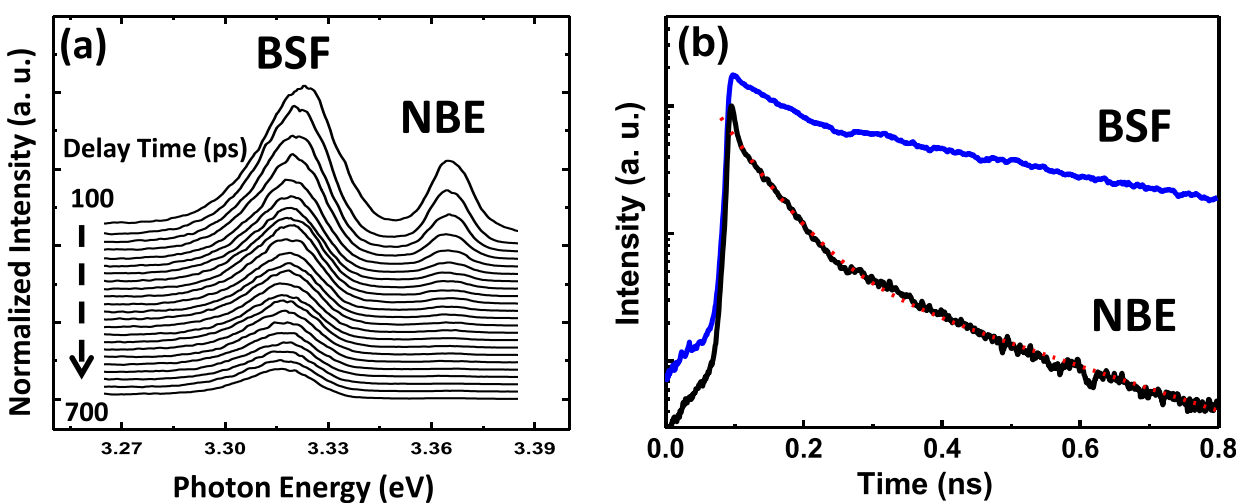

FIG. 2. (a) TR-PL spectra with time delays in steps of 100 ps after the laser pulse measured at $5 \mathrm{~K}$ with an excitation power of $100 \mu \mathrm{W}$. Each curve has the integrated time of $25 \mathrm{ps}$, and the time interval between each other is 25 ps. (b) The decay curves of the intensity integrated from the BSF and NBE emissions in energy ranges of $3.280-3.345 \mathrm{eV}$ and $3.345-3.397 \mathrm{eV}$, respectively. The decay curve at the bottom is the response of the instrument.
Figure 1(d), the peak energy of the FX transition as a function of temperature is fitted to Varshni's formula, which is shown as a red dashed line and determines the transition energy at $0 \mathrm{~K}$ to be about $3.375 \mathrm{eV}$. The FX tends to be bound by a donor to form $\mathrm{D}^{\circ} \mathrm{X}$ below $100 \mathrm{~K}$, which is consistent with previous observations. ${ }^{29}$ To confirm this assumption, we performed TRPL measurements. In Figure 2(a), the TR-PL spectra at a temperature of $5 \mathrm{~K}$ are monitored after a pulsed excitation. With increasing time after the pulse, the BSF emission band shows a redshift from 3.322 to $3.315 \mathrm{eV}$, while the NBE emission band remains at about $3.362 \mathrm{eV}$. Such an observed behavior is a typical feature for a relaxation process of the BSF-bound electrons in the band tail states. ${ }^{30}$ To determine the average decay times of the BSF and NBE emission bands, the decay curves are plotted in Figure 2(b). The decay curve of the BSF (NBE) emission band is obtained by integrating the intensity in the energy range from 3.280 to $3.345 \mathrm{eV}$ (from 3.345 to $3.397 \mathrm{eV}$ ). Each decay curve is fitted by a bi-exponential function: $I(t)=A_{1} \exp \left(-t / \tau_{1}\right)+A_{2} \exp \left(-t / \tau_{2}\right)$. For the NBE emission, the decay times are determined to be $\tau_{1} \approx 52 \mathrm{ps}, \tau_{2} \approx 250$ ps, and the ratio of $A_{1} / A_{2}$ is about 8.3 , which means that $\tau_{1}$ is the decay time of the NBE in which the $\mathrm{D}^{\circ} \mathrm{X}$ transition dominates. The decay time of the NBE band agrees with the reported lifetime of $\mathrm{D}^{\circ} \mathrm{X}$, and $\tau_{2}$ is the decay time resulting from the free exciton transition. ${ }^{31,32}$ For the BSF emission, the decay times are $\tau_{1} \approx 55 \mathrm{ps}$ and $\tau_{2} \approx 450 \mathrm{ps}$. Corfdir et al. reported the decay curves of the emissions in BSFs of the GaN a-plane films. ${ }^{33}$ They found that bi-exponential decay curves only occur in the region of the BSFs bundles. The origin of the shorter decay time $\tau_{1}$ is attributed to the complex overlapping of electron and hole wave function in the different distributions of inter-BSF distances. The longer decay time $\tau_{2}$ is due to the radiative recombination of the $\mathrm{BSF}-$ bound electrons with the holes in the $\mathrm{W}-\mathrm{ZnO} .^{26}$ Using the rate equation analysis, ${ }^{34}$ the rate equations of the transitions of $\mathrm{FX}$-to- $\mathrm{D}^{0} \mathrm{X}$ and $\mathrm{FX}$-to-BSF could be written as follows:

$$
\begin{gathered}
d n_{D X} / d t=n_{F E} / \tau_{F E \rightarrow D X}-n_{D X} / \tau_{D X}, \\
d n_{B S F} / d t=n_{F E} / \tau_{F E \rightarrow B S F}-n_{B S F} / \tau_{B S F},
\end{gathered}
$$

where $n_{F E}$ is the density of the free excitons, $\tau_{F E}$ is the lifetime of the free exciton, and the constants $\tau_{F E \rightarrow D X}$ and $\tau_{F E \rightarrow B S F}$ are the lifetimes of the capture transitions of FX-to$\mathrm{D}^{0} \mathrm{X}$ and FX-to-BSF, respectively. In the steady state, the $d n_{D X} / d t$ and $d n_{B S F} / d t$ in the rate equations are zero. Besides, the intensities of $\mathrm{FX}, \mathrm{D}^{0} \mathrm{X}$, and $\mathrm{BSF}$ are presented as $I_{F E}$
$=n_{F E} / \tau_{F E}, I_{D X}=n_{D X} / \tau_{D X}$, and $I_{B S F}=n_{B S F} / \tau_{B S F}$. Therefore, we could obtain the lifetimes of the capture transitions of FXto-D ${ }^{0} \mathrm{X}\left(\tau_{F E \rightarrow D X}\right)$ and FX-to-BSF $\left(\tau_{F E \rightarrow B S F}\right)$ from Eqs. (3) and (4)

$$
\begin{aligned}
\tau_{F E \rightarrow D X} & =I_{F E} \tau_{F E} / I_{D X}, \\
\tau_{F E \rightarrow B S F} & =I_{F E} \tau_{F E} / I_{B S F} .
\end{aligned}
$$

Based on the PL results, the intensity ratio of $\mathrm{D}^{0} \mathrm{X}$ to $\mathrm{FE}$ is about 8.3 and the lifetime of the FX is $\sim 250$ ps. Therefore, the value of $\tau_{F E \rightarrow D X}$ can be roughly estimated to be about 6 ps. Similarly, the intensity ratio of BSF to FX is about $1.67 \times 10^{4}$, so the value of $\tau_{F E \rightarrow B S F}$ can be roughly estimated to be $15 \mathrm{fs}$.

To gain more information on the details of the band tails, spectrally dependent decay times are plotted. In Figure 3 , the PL decay times at different monitored emission energies are plotted together with the corresponding TI-PL spectra (solid curves). The decay times of the BSF emission gradually increase with decreasing emission energy, while the NBE emission appears to have fixed decay times of the $\mathrm{D}^{\circ} \mathrm{X}$ transition for an excitation power of $100 \mu \mathrm{W}$ in Figure 3(a). Such a significant difference implies that carriers migrating among band tail states will occur for the BSFs. In the energy range of the BSF emission band, the density of band tail states is estimated to follow $\exp \left(-E / E_{o}\right)$, and the radiative recombination time $\tau_{r}$ as a constant does not vary with altered emission energies. The decay time $\tau(E)$ can be expressed as ${ }^{30,35}$

$$
\tau(E)=\frac{\tau_{r}}{1+\exp \left[\left(E-E_{m e}\right) / E_{o}\right]},
$$

where $E_{m e}$ is the characteristic energy, which plays a role analogous to that of a mobility edge, and the inverse value of the radiative recombination time corresponds to the transfer rate for electrons from the band tail states to the valance band. The parameter $E_{o}$ represents the characteristic energy of the density distribution of the band tail states, which also plays a role analogous to the depth of the band tail state. ${ }^{31,36}$ Spectrally dependent decay time curves with excitation powers from 10 to $1020 \mu \mathrm{W}$ at $5 \mathrm{~K}$ (not shown here) reveal an ambiguous cross at $\sim 3.33 \mathrm{eV}$. This observation implies that the value of $E_{m e}$ is consistent with $3.33 \mathrm{eV}$, evaluated from Eq. (4). For the case of an excitation laser power of $100 \mu \mathrm{W}$, the best fits in Figure 3(a) are $\tau_{r}=758 \mathrm{ps,}$ $E_{m e}=3.33 \mathrm{eV}$, and $E_{0}=18 \mathrm{meV}$. As the temperature is 


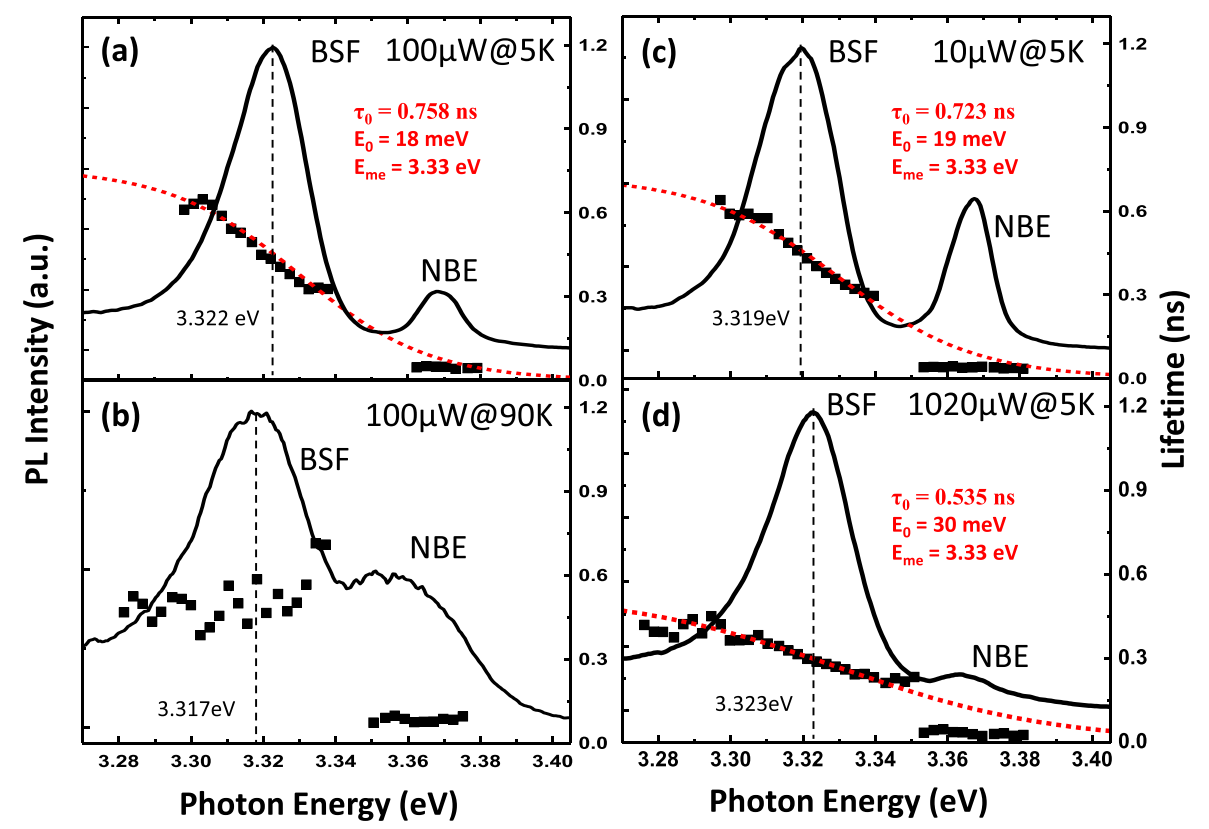

FIG. 3. The spectrally dependent decay times are plotted with the same excitation power, $100 \mu \mathrm{W}$, at different temperatures; (a) 5 and (b) $90 \mathrm{~K}$, and with different excitation power; (c) 10 and (d) $1020 \mu \mathrm{W}$ at the same temperature $5 \mathrm{~K}$. raised to $90 \mathrm{~K}$, the curve of the decay times versus the monitored photon energy (in Fig. 3(b)) in the BSF emission band reveals an almost constant value of about $450 \mathrm{ps}$. Such result indicates that the BSF-bound excitons are delocalized from the band tail states by thermal excitation at $90 \mathrm{~K}$. Further, we performed TR-PL with excitation powers of $10 \mu \mathrm{W}$ and $1020 \mu \mathrm{W}$ at $5 \mathrm{~K}$, and the decay times versus the photon energy are shown in Figures 3(c) and 3(d). The best fitting results yield $\tau_{r}=723 \mathrm{ps}(535 \mathrm{ps})$ and $E_{o}=19 \mathrm{meV}(30 \mathrm{meV})$ for an excitation power of $10 \mu \mathrm{W}(1020 \mu \mathrm{W})$. The band profile of the QWs structure grown along the polar axis should be tilted by the internal electric field that leads to QWs of triangular shape, which is in agreement with the results of power-dependent TI-PL. Therefore, a high density of excited carriers is produced by the high excitation power, and they accumulate at the interface between the potential well and barrier due to the Coulomb attraction, which results in a band bending effect that raises the electron quantization energy as mentioned above and enhances the overlap of the wave functions of the electron and the hole. Such an enhancement of the wave function overlap is in agreement with the decrease of the radiative recombination time $\tau_{r}$ at high excitation power in Figure 3(d). Besides, the electron quantization energy level increases, which means an increased separation between the energy levels among the band tails states followed by a broadening of the distribution. This fact raises the value of $E_{o}$, as observed in Figure 3(d). The separation between the energy levels among the band tail states is also considered to increase with high excitation laser power due to the band bending effect, which results in a high density of photon-excited carriers.

In conclusion, we have studied BSFs-related localized carrier recombination dynamics in the $\mathrm{m}$-plane $\mathrm{ZnO}$ epitaxial films. The randomly distributed type-II QWs are formed by the high density of BSFs in the $\mathrm{ZnO}$ materials, leading to a wave function coupling between the BSF-bound electrons. By TI-PL and TR-PL measurements, which represent the carrier dynamics in the BSFs, reveal the migration behavior of the BSF-bound excitons in the band tails states. High excitation laser power leads to a band bending effect, which in turn broadens the distribution of the band tail states, and subsequently increases the depth of the distribution as well as causing an energy shift of the emission band due to the type-II QWs.

The authors are thankful to P. Bergman in IFM for providing the time-resolved facilities. This work was partly supported by National Science Council of Taiwan under Grant Nos. NSC-99-2112-M-006-017-MY3, NSC-100-2112M-213-002-MY3, and NSC-102-2112-M-006-012-MY3.

${ }^{1}$ Ü. Özgür, Ya. I. Alivov, C. Liu, A. Teke, M. A. Reshchikov, S. Doğan, V. Avrutin, S.-J. Cho, and H. Morkoç, J. Appl. Phys. 98, 041301 (2005).

${ }^{2}$ D. C. Look, D. C. Reynolds, C. W. Litton, R. L. Jones, D. B. Eason, and G. Cantwell, Appl. Phys. Lett. 81, 1830 (2002).

${ }^{3}$ T. Wang, H. Wu, H. Zheng, J. B. Wang, Z. Wang, C. Chen, Y. Xu, and C. Liu, Appl. Phys. Lett. 102, 141912 (2013).

${ }^{4}$ W.-R. Liu, Y.-H. Li, W. F. Hsieh, W. C. Lee, M. Hong, J. Kwo, and C.-H. Hsu, J. Phys. D: Appl. Phys. 41, 65105 (2008).

${ }^{5}$ R. Ravash, P. Veit, M. Müller, G. Schmidt, A. Dempewolf, T. Hempel, J. Bläsing, F. Bertram, A. Dadgar, J. Christen, and A. Krost, Phys. Status Solidi C 9, 507 (2012).

${ }^{6}$ S. Yang, C. C. Kuo, W.-R. Liu, B. H. Lin, H. C. Hsu, C. H. Hsu, and W. F. Hsieh, Appl. Phys. Lett. 100, 101907 (2012).

${ }^{7}$ W. H. Lin, U. Jahn, H. T. Grahn, L. Chang, M. M. C. Chou, and J. J. Wu, Appl. Phys. Express 6, 061101 (2013).

${ }^{8}$ W. Rieger, R. Dimitrov, D. Brunner, E. Rohrer, O. Ambacher, and M. Stutzmann, Phys. Rev. B. 54, 17596 (1996).

${ }^{9}$ P. Corfdir, P. Lefebvre, J. Levrat, A. Dussaigne, J.-D. Ganiere, D. Martin, J. Ristic, T. Zhu, N. Grandjean, and B. Deveaud-Pledran, J. Appl. Phys. 105, 043102 (2009).

${ }^{10}$ Y. J. Sun, O. Brandt, U. Jahn, T. Y. Liu, A. Trampert, S. Cronenberg, S. Dhar, and K. H. Ploog, J. Appl. Phys. 92, 5714 (2002).

${ }^{11}$ Y. Yan, G. M. Dalpian, M. M. Al-Jassim, and S.-H. Wei, Phys. Rev. B. 70, 193206 (2004).

${ }^{12}$ Y. Zhang, L.-W. Wang, and A. Mascarenhas, Nano Lett. 7, 1264 (2007).

${ }^{13}$ J. Schrier, D. O. Demchenko, L.-W. Wang, and A. P. Alivisatos, Nano Lett. 7, 2377 (2007).

${ }^{14}$ S. Yang, B. H. Lin, C. C. Kuo, H. C. Hsu, W.-R. Liu, M. O. Eriksson, P.O. Holtz, C.-S. Chang, C.-H. Hsu, and W. F. Hsieh, Cryst. Growth Des. 12, 4745 (2012).

${ }^{15}$ W.-R. Liu, W. F. Hsieh, C.-H. Hsu, K. S. Liang, and F. S.-S. Chien, J. Appl. Cryst. 40, 924 (2007).

${ }^{16}$ W.-R. Liu, Y.-H. Li, W. F. Hsieh, C.-H. Hsu, W. C. Lee, Y. J. Lee, M. Hong, and J. Kwo, Cryst. Growth Des. 9, 239 (2009). 
${ }^{17}$ M. R. Wagner, G. Callsen, J. S. Reparaz, J.-H. Schulze, R. Kirste, M. Cobet, I. A. Ostapenko, S. Rodt, C. Nenstiel, M. Kaiser, A. Hoffmann, A. V. Rodina, M. R. Phillips, S. Lautenschläger, S. Eisermann, and B. K. Meyer, Phys. Rev. B 84, 035313 (2011).

${ }^{18}$ A. Zeuner, H. Alves, D. M. Hofmann, B. K. Meyer, M. Heuken, J. Biasing, and A. Krost, Appl. Phys. Lett. 80, 2078 (2002).

${ }^{19}$ Y. Chen, H. J. Ko, S. K. Hong, and T. Yao, Appl. Phys. Lett 76, 559 (2000).

${ }^{20}$ S.-Y. Lin, C.-C. Tseng, W.-H. Lin, S.-C. Mai, S.-Y. Wu, S.-H. Chen, and J.-I. Chyi, Appl. Phys. Lett. 96, 123503 (2010).

${ }^{21}$ T. Kawazu, T. Mano, T. Noda, and H. Sakaki, Appl. Phys. Lett. 94, 081911 (2009).

${ }^{22}$ T. Kuroda and A. Tackeuchi, J. Appl. Phys. 92, 3071 (2002).

${ }^{23}$ S. F. Chichibu, A. C. Abare, M. P. Mack, M. S. Minsky, T. Deguchi, D. Cohen, P. Kozodoy, S. B. Fleischer, S. Keller, J. S. Speck, J. E. Bowers, E. Hu, U. K. Mishra, L. A. Coldren, S. P. DenBaars, K. Wada, T. Sota, and S. Nakamura, Mater. Sci. Eng. B 59, 298 (1999).

${ }^{24}$ T. T. Chen, C. L. Cheng, Y. F. Chen, F. Y. Chang, H. H. Lin, C.-T. Wu, and C.-H. Chen, Phys. Rev. B. 75, 033310 (2007).

${ }^{25}$ S. D. Singh, V. K. Dixit, S. Porwal, R. Kumar, A. K. Srivastava, T. Ganguli, T. K. Sharma, and S. M. Oak, Appl. Phys. Lett. 97, 111912 (2010).

${ }^{26}$ D. Alonso-Álvarez, B. Alén, J. M. Gercía, and J. M. Ripalda, Appl. Phys. Lett. 91, 263103 (2007).
${ }^{27}$ Y.-H. Cho, G. H. Gainer, J. B. Lam, and J. J. Song, Phys. Rev. B. 61, 7203 (2000).

${ }^{28}$ P. G. Eliseev, P. Perlin, J. Lee, and M. Osiński, Appl. Phys. Lett. 71, 569 (1997).

${ }^{29}$ V. A. Fonoberov, K. A. Alim, A. A. Balandin, F. Xiu, and J. Liu, Phys. Rev. B. 73, 165317 (2006).

${ }^{30}$ C. Gourdon and P. Lavallard, Phys. Status Solidi B 153, 641 (1989).

${ }^{31}$ Y. Narukawa, Y. Kawakami, S. Fujita, and S. Fujita, Phys. Rev. B 55, R1938 (1997).

${ }^{32}$ S. F. Chichibu, T. Onuma, M. Kubota, and A. Uedono, J. Appl. Phys. 99, 093505 (2006).

${ }^{33}$ P. Corfdir, J. Ristic, P. Lefebvre, T. Zhu, D. Martin, A. Dussaigne, J. D. Ganiere, N. Grandjean, and B. Deveaud-Pledran, Appl. Phys. Lett. 94, 201115 (2009).

${ }^{34}$ T. Schmidt, K. Lischka, and W. Zulehner, Phys. Rev. B 45, 8989 (1992).

${ }^{35}$ M. Strassburg, M. Dworzak, H. Born, R. Heitz, A. Hoffmann, M. Barteis, K. Lischka, D. Schikora, and J. Christen, Appl. Phys. Lett. 80, 473 (2002).

${ }^{36}$ I. A. Buyanova, J. P. Bergman, G. Pozina, W. M. Chen, S. Rawal, D. P. Norton, S. J. Pearton, A. Osinsky, and J. W. Dong, Appl. Phys. Lett. 90, 261907 (2007). 